\title{
A Velocity-Modulation Television System
}

$\mathrm{M}$ ANY of the investigators who are seeking at the present time to develop a practical system of television make use of the cathode ray oscillograph tube in one form or another, since the electron beam in such a tube provides an easily controlled means of scanning the picture to be transmitted. At the receiving end, the cathode ray tube is employed to build-up the received picture by varying the intensity of the beam in accordance with the light and dark portions of the picture. The ordinary type of cathode ray tube, however, gives only a small range of intensity control without the accompaniment of loss of focus of the spot on the fluorescent screen, and special electrode systems have to be arranged to obtain good intensity modulation in this manner. As an alternative to this method, the intensity of the cathode ray beam may be kept constant but its tranverse velocity may be varied as it moves over the picture, the beam being speeded up over the dark portions of the picture and slowed down over the light portions. The corresponding motion of the cathode ray beam at the receiving end thus gives varying illumination according to the speed of travel of the spot on the fluorescent screen, and with the aid of the phenomenon of persistence of vision, a true impression of the shades and contrasts in the picture received is obtained.

The conception of this velocity-modulation principle, or variable-speed cathode ray television, dates back to 1911, when it was described in a British patent by B. Rosing. Since that date the principle appears to have fallen into oblivion until it was revived in Germany by $R$. Thun in 1929 . The first practical realisation of the method was achieved by $M$. von Ardenne in 1931 and reference was made to this work in NATURE of October 7 last (p. 573).

During the development of cathode ray oscillograph tubes for general scientific and technical purposes, the staff of Messrs. A. C. Cossor Ltd. realised the possibilities of the above system of television, and an account of the development work carried out during the past eighteen months was presented in a paper entitled "A Velocity-Modulation Television System", read before the Wireless Section of the Institution of Electrical Engineers by Messrs. L. H. Bedford and O. S. Puckle on February 7.

Consideration of the basic principles outlined above shows that it is impossible to realise a velocitymodulated picture from a uniformly scanned object; the scanning at the transmitter must also be of the variable-speed or velocity-modulated type, and must therefore be carried out by a cathode ray. It follows that a cathode ray oscillograph must serve as the source of light at the transmitting end, and, with oscillographs of the ordinary low-voltage type, the conditions of scanning-light economy will restrict the picture subject matter to cinematograph film material. This, howerer, is not considered to be a disadvantage of the method; many of the television systems being developed at the present time make use of a film as an intermediary, and processes are being devised in which the interval between the photography of the subject and the projection of the picture through the transmitter is reduced to the bare minimum.

The transmitting arrangements described by
Messrs. Bedford and Puckle comprise the projection of light from the fluorescent screen of the oscillograph through the film picture on to a photoelectric cell. The output of the photo-cell amplifier operates, through a screen-grid valve and a thyratron, an electrical time-base circuit which supplies the potential difference to one pair of the deflecting plates of the oscillograph. The light from the cathode ray tube is thus swept in a straight line across the picture with a velocity which varies according to its transparency at different points. At the end of each scanning line, the discharge of the thyratron provides a 'fly-back' action to the spot sufficiently rapid to be invisible. Simultaneously with this operation, a second valve and thyratron circuit provides a traversing time-base potential difference to the second pair of deflecting plates of the oscillograph tube. By this means the scanning line is traversed across the picture in successive steps.

From this description it will be realised that an image of the picture being transmitted is built up on the fluorescent screen of the cathode ray oscillograph, and this is found to be a useful feature of the system for monitoring purposes. Furthermore, for the reproduction of the image on the screen of another oscillograph tube at a distant receiving station, it is merely necessary to transmit to the second tube the voltages being applied to the two pairs of deflecting plates of the first tube. If these voltages are sent through two separate channels, the received picture is automatically synchronised with that at the transmitting end.

The authors of the paper referred to above have modified this arrangement to some extent, however, to enable all the intelligence to be sent along a single channel. Using a picture frequency of 25 per second with a detail corresponding to 120 or 160 scanning lines, the transmitted signals require a frequency band of the order of 240 kilocycles per second; and special amplifiers have been developed to give uniform amplification over this range. The size of the picture received depends upon the deflector voltages which may be applied to the oscillograph electrodes, and it is anticipated that future design and manufacture will enable a suitable receiver tube with a 9-inch screen to be produced. Among the advantages of the method described above over that employing intensity-modulation are the increased picture brightness for a given receiving oscillograph and the concentration of detail in the light portions of the picture.

Although Messrs. Bedford and Puckle's experiments have so far been limited to transmission over wire lines, no particular difficulty is anticipated in applying the necessary signals to radio transmission, at least on the ultra-short wave-length of a few metres where such a large frequency band as $\mathbf{2 4 0}$ kc./sec. may be permitted. At the reading of the paper, a cinematograph film was shown illustrating typical pictures received in a laboratory test of the whole system. Among the features brought out in this demonstration was the fact that, when required to obtain a better contrast ratio in the received picture, intensity modulation may be superimposed with advantage upon the velocity-modulation signals, and means of achieving this very satisfactory combination are being investigated. 